$4 \mid 2017$

Technologies numériques et diffusion de l'information pendant les périodes de conflits et de crise dans le monde

\title{
De la bataille des chiffres à la bataille des mots. Tensions autour de la fabrication d'une crise
}

A battle over numbers, a battle over words: tensions around the construction of a crisis

Streit um zahlen, streit um worte: die konstruktion einer krise

Jean-Marie Stratigos

\section{OpenEdition}

\section{Journals}

Édition électronique

URL : http://journals.openedition.org/ctd/823

DOI : $10.4000 /$ ctd. 823

ISSN : 2491-1437

Éditeur

Chaire Unesco Pratiques émergentes en technologies et communication pour le développement

Référence électronique

Jean-Marie Stratigos, « De la bataille des chiffres à la bataille des mots. Tensions autour de la

fabrication d'une crise », Communication, technologies et développement [En ligne], 4| 2017, mis en ligne le 04 septembre 2017, consulté le 29 mars 2021. URL : http://journals.openedition.org/ctd/823 ; DOI : https://doi.org/10.4000/ctd.823

Ce document a été généré automatiquement le 29 mars 2021.

Communication, technologies et développement 


\title{
De la bataille des chiffres à la bataille des mots. Tensions autour de la fabrication d'une crise
}

\author{
A battle over numbers, a battle over words : tensions around the construction of \\ a crisis
}

Streit um zahlen, streit um worte: die konstruktion einer krise

Jean-Marie Stratigos

\section{Du phénomène naturel au désastre humanitaire}

1 Le 17 février 2008 à l'aube, le cyclone Ivan frappe la côte Est de Madagascar puis traverse la Grande Île avant de se dissoudre dans le golfe du Mozambique, laissant dans son sillage désolation et destruction. Depuis le 5 février, les membres de la configuration humanitaire ${ }^{1}$ à Madagascar, réunis au sein du Comité Restreint des Intervenants dans les Catastrophes (CRIC), suivaient anxieusement l'évolution d'une dépression apparue au nord de l'Ile de la Réunion, soit moins d'une semaine après qu'un premier cyclone dénommé Fame n'ait atteint le pays. Progressivement, la dépression gagne en puissance. Le 7 février, elle devient une dépression tropicale, puis une tempête tropicale le 9 février quand ses vents dépassent $63 \mathrm{Km} /$ heure. Elle reçoit alors le nom d'Ivan tandis qu'elle continue de se renforcer et de se rapprocher de Madagascar. Le 16 février, ses vents atteignent $231 \mathrm{Km} /$ heure et elle change de genre pour devenir un cyclone ${ }^{2}$ de catégorie 3 sur l'échelle de Saffir-Simpson.

Dès qu'il atteint la côte, le Bureau National de Gestion des Risques et des Catastrophes (BNGRC), l'entité gouvernementale chargée de la préparation et de la réponse aux urgences, émet un premier communiqué officiel. Il est question de 83 morts, de 177 disparus et de plus de 187000 sans- abri. Rapidement, les partenaires humanitaires commencent à relayer l'information dans leurs bulletins internes et externes. C'est ensuite au tour de la presse nationale de faire ses gros titres sur l'événement. L'hebdo de 
Madagascar (22 février 2008) consacre son édition aux dégâts occasionnés et barre sa une d'un "Cyclone Ivan le Terrible». Finalement, l'information se diffuse hors de Madagascar et devient globale.

Dans tous les médias le passage d'Ivan est qualifié de catastrophe naturelle. Cependant, il convient de rappeler que depuis les conclusions de la « Décennie Internationale pour la Réduction des Désastres Naturels » (1990-1999) il est d'usage de mettre le terme naturel entre guillemets afin de rappeler qu'un cyclone est un événement normal et que ce n'est que lorsqu'il entre en contact avec l'activité humaine qu'il devient un « désastre ( (Oliver Smith 1996 : 303).

Ce n'est donc que par l'intermédiaire de systèmes classificatoires humains qu'un événement naturel s'est vu labellisé cyclone, puis désastre, et a été baptisé Ivan. Ce nom permet même de le lui associer des qualités humaines comme L'hebdo de Madagascar qui fait une analogie avec le tsar Ivan le Terrible, réputé pour sa violence et sa cruauté. Mais ce processus de labellisation (Escobar $1991: 667$, Wedel $2005: 37$ ) ne se limite pas à l'événement climatique. Tout ce qui croise son chemin et participe de la catastrophe émerge sous une nouvelle dénomination. Les villages, rizières et les bateaux de pêche deviennent des dégâts qui deviendront à leur tour des projets. Les hommes et femmes affectés deviennent des victimes qui seront bientôt des bénéficiaires. Tel le Dieu de la Bible créant le monde par le Verbe, les membres de la configuration humanitaire se transforment en démiurges modernes et créent la réalité de la crise.

Cette fabrication de la crise repose sur l'information, depuis sa collecte jusqu'à sa diffusion. Une population affectée par le passage d'un cyclone, sans que l'événement ne soit quantifié et qualifié, ne se transforme ni en « victime » ni en "bénéficiaire ». Sans témoignage, les solidarités ne peuvent être mobilisées et aucune contribution ne se matérialise pour financer les programmes d'aide. Sans ce processus de création par l'information, la crise n'existe pas, et l'activité humanitaire non plus. Ce processus initial est donc essentiel dans le cycle humanitaire; c'est pourquoi la collecte de l'information, sa gestion, sa mise en forme et sa diffusion sont les enjeux de tensions et de compétitions entre les différents membres de la configuration humanitaire.

\section{Méthodologie}

6 Le propos de cet article est de mettre en évidence ces tensions ainsi que l'importance de la maîtrise des technologies de l'information et des techniques de la communication dans cette compétition. Il est basé sur un travail ethnographique et anthropologique mené en 2007 et 2008 à Madagascar et couvre l'ensemble de la "saison cyclonique ». Il convient de noter que la notion de saison cyclonique est utilisée aussi bien par la communauté humanitaire que par les services météorologiques malgaches. Pour ces derniers, elle définit la période située entre novembre et avril durant laquelle les conditions sont remplies pour que des tempêtes se créent sur l'océan Indien, et pour que certaines évoluent en cyclones qui peuvent affecter le pays. Pour les humanitaires, elle constitue la ligne directrice de leur action sur douze mois, en amont comme en aval. Elle est aussi et surtout la justification à leur présence. La « saison cyclonique » est pour eux un terme émique qui recouvre un sens plus large que son exposé, et un concept opératoire central à leur action. Cette recherche a été menée en tant que responsable du Bureau de Coordination des Affaires Humanitaires des Nations Unies $(\mathrm{OCHA})^{3}$ à Madagascar donc dans la posture de l'observation participante. Ce rôle de 
coordinateur de l'action humanitaire procurait un accès privilégié aux différents acteurs impliqués, socialement et professionnellement. Il conférait aussi la légitimité nécessaire pour interagir avec chacun d'eux; employés des Nations Unies, des ONG locales et nationales, représentants de l'état et de la société civile, ainsi que les bailleurs de fonds. Il est important de souligner que la position de chef du bureau OCHA, du fait de sa position hiérarchique, se trouve dans les lieux de pouvoir, et non au contact des bénéficiaires de l'aide.

7 Pour finir, il convient de préciser que Madagascar a été le cadre de l'étude, pas son objet. Comme le rappelle Geertz (1998 : 94) l'anthropologue n'étudie pas des lieux, mais des interactions humaines qui prennent place dans ces lieux. Dans le cadre de ce travail, l'objet était la configuration humanitaire malgache, ainsi que les interactions entre ses membres, durant un événement déterminant pour le groupe et pour chacun d'eux : l'arrivée du cyclone Ivan en février 2008. L'analyse de la presse locale se place dans la démarche de Simonin (1999: 93) avec le souci d'envisager les journaux étudiés «dans leur diversité (et leurs) modalités de mises en forme événementielles » et sur la base d'une sélection représentative des différentes sensibilités politiques de la scène malgache. Du fait de contraintes matérielles et temporelles, cette analyse ne couvre pas les nouvelles technologies de l'information et de la communication (NTIC) qui auraient aussi pu être utilisées pour rendre compte de la différence de traitement de l'événement (médias en ligne, médias sociaux, médias audiovisuels et télévisuels).

Nous observerons dans cet article comment le contrôle de l'information autour de l'événement cyclonique revêt des attentes et des symboliques différentes chez les humanitaires étrangers et leurs contreparties nationales. Outil d'indépendance et de renforcement du récit national pour le Gouvernement, il est plus prosaïquement un moyen de levées de fonds pour les organisations internationales. Cependant, il apparaîtra qu'au delà de ces différences, les parties en présence s'affrontent aussi pour la captation et le contrôle de la manne humanitaire. Nous rappellerons enfin l'enracinement des techniques de l'information dans le paradigme occidental et comment cette filiation donne un avantage aux partenaires du Nord dans cet exercice de construction de la réalité. Pour ce faire, nous découvrirons comment la collecte de données suite au passage d'un cyclone, ainsi que leur mise en récit et leur diffusion, se traduisent par une bataille des chiffres et une bataille des mots qui au final affectent négativement la relation entre les membres de la configuration humanitaire.

\section{La collecte et la gestion des données : la bataille des chiffres}

9 La gestion de la précédente saison cyclonique (2006-2007) est unanimement considérée comme l'une des pires au niveau mondial. C'est pourquoi les sièges des Agences des Nations Unies à New York, comme les Bureaux Régionaux à Johanesbourg supervisent attentivement la situation à Madagascar et ont renforcé leurs équipes sur le terrain. Un des problèmes majeurs durant la saison cyclonique précédente a été la pléthore de données contradictoires collectées et diffusées. Le Gouvernement estimait les dégâts causés à 252 millions de dollars tandis que les partenaires étrangers les évaluaient à $10 \%$ de cette somme. Face à ce désaccord, les pays donateurs n'avaient financé que $5 \%$ de l'appel du Gouvernement (La Gazette 22 février 2008), le rejetant virtuellement. Or formuler une demande et la voir refusée est considéré comme une humiliation à 
Madagascar (Keenan et Ochs $1979: 154)$. C'est donc avec une certaine fébrilité que les partenaires s'engagent dans cette nouvelle crise.

\section{Systèmes d'alerte précoce et gestion des données}

10 Avant même qu'un désastre survienne, les systèmes d'alerte précoce doivent permettre de l'anticiper et de modérer son impact. A Madagascar plusieurs systèmes sont en place, fruits de la non- coordination des efforts de développement et témoins de la compétition entre pays donateurs. Ils sont parfois complémentaires, parfois concurrents. On trouve ainsi le Système d'Alerte Précoce (SAP), le système National d'Alerte Précoce (SNAP), le réseau d'observatoires ruraux (ROR), l'observatoire du Riz (ODR) et le Système d'information rural de la sécurité alimentaire (SIRSA). De plus, le Programme Alimentaire Mondial (PAM) gère une unité d'" Analyse et Cartographie de la Vulnérabilité à l'Insécurité Alimentaire " (ACV). Finalement, on trouve le National Vulnerabilty Assessment Committee (NVAC), prévu dans le cadre d'accords régionaux, qui doit être mis en place par le PAM.

L'établissement d'un système permettant de collecter et de traiter les données relatives aux crises à venir est vu comme une priorité absolue par les partenaires internationaux et leur préférence va vers le SAP qui est pleinement fonctionnel. Or, le gouvernement a refusé de prendre en charge ce système que l'Union européenne souhaite lui transférer après l'avoir financé pendant 10 ans. Au contraire, il lui privilégie le développement du Système Nationale d'Alerte Précoce (SNAP). Ce dernier, qui a été développé pendant huit ans par ajouts successifs, n'est toujours pas fonctionnel et ses fondements sont irréalistes. Il prévoit par exemple de collecter les données en réinstaurant le réseau de coureurs de l'ère monarchique qui transportait à travers le pays des dépêches dans des cylindres de bambou et de le coupler avec un réseau de PDA (Personal Digital Assistant) alors que la couverture numérique est inexistante hors des villes. D'autre part il propose d'utiliser des indicateurs d'évaluation de vulnérabilité tels que le nombre de femmes portant un lambaoany (vêtement) déchiré au point d'eau ou le nombre de pavillons loués par le vendeur de confection et de friperies, indicateurs qui ne convainquent pas les partenaires étrangers.

12 Pour le Gouvernement et le Bureau National de Gestion des Risques et des Catastrophes, le SNAP est un outil d'indépendance et de fierté nationale et ils lui apportent un soutien indéfectible. Il possède dans son intitulé le « $\mathrm{N}$ », comme National, en opposition au SAP qui est perçu comme un outil développé par les étrangers et que l'Union européenne tenterait d'imposer. Pour les partenaires internationaux en revanche, il s'agit d'un choix technique, destiné à optimiser les collectes de fonds. Mais cela s'apparente aussi même s'ils ne le conçoivent pas ainsi - à une démarche paternaliste: ils souhaitent contrôler les flux de données pendant la crise, et, en conséquence la répartition de l'aide internationale d'urgence.

13 Pour les partenaires internationaux, le contrôle de la gestion des données au niveau local ne constitue qu'une étape dans un système plus large. En effet, l'information produite au niveau local est ensuite transférée à un réseau régional et global de bases de données. Le Bureau OCHA à Johannesbourg gère ainsi un système d'information régionale appelé «Southern African Humanitarian Information Management Network» ou SAHIM, et le siège de l'Agence à Genève gère le site Reliefweb ${ }^{4}$ qui dissémine de l'information sur l'ensemble des crises au niveau mondial. 
Il est essentiel, pour que ces réseaux fonctionnent, qu'ils soient alimentés en données primaires par les terrains d'opération. Ceci explique que les urgentistes tentent de s'assurer, sinon du contrôle nominal de la base de données locales, de l'existence d'un système qui répond à leurs attentes et à leurs standards. Ils ignorent, ou veulent ignorer que leur action s'intègre à un jeu politique local: Le Gouvernement veut affirmer l'indépendance et la fierté nationale, le Bureau National de Gestion des Risques et des Catastrophes (BNGRC) souhaite utiliser le système de base de données comme un élément d'émancipation de ses partenaires internationaux et le Secrétaire Exécutif du BNGRC souhaite s'approprier le Système National d'Alerte Précoce comme un outil de sa propre promotion.

SAP ou SNAP, SAHIM ou Reliefweb, quels que soit les systèmes retenus et utilisés, il est nécessaire qu'ils soient alimentés par des données pour remplir leur rôle. Pour ce faire, des mécanismes de collectes de données existent, qui doivent permettre de quantifier et de qualifier les dégâts occasionnés par le passage du cyclone et de définir les besoins des populations concernées. Cette phase de collecte est, elle aussi, affectée par des tensions antagonistes entre les partenaires.

\section{Planification de contingence et collecte des données}

16 Le plan d'urgence, mis au point par les partenaires du Comité Restreint des Intervenants dans les Catastrophes (CRIC) durant l'automne 2007, détaille les processus de collectes de données qui doivent permettre de disposer d'informations sur la catastrophe immédiatement après qu'elle se soit produite. Les deux axes principaux sont : l'envoi d'équipes d'experts vers les zones affectées depuis la capitale pour des évaluations rapides, et la remontée d'information du terrain grâce aux fiches EIMA (Enquête Initiale Multi Aléas, un questionnaire utilisé depuis plusieurs années à Madagascar) envoyées par les autorités locales. Ces mécanismes impliquent l'ensemble des partenaires humanitaires; partenaires internationaux et nationaux, publiques et ONG, et le Secrétaire Exécutif du Bureau National de Gestion des Risques et des Catastrophes (BNGRC) insistent dans la lettre de lancement du "plan de contingence » sur le caractère collectif de ce document.

17 Ce plan d'urgence (pp.33-34) détaille dans les moindres détails les modalités de ces collectes pour chaque secteur: les formulaires à utiliser, le nom des personnes qui doivent y participer, ainsi que les modes de transport. Cependant, après le passage du premier cyclone Fame en janvier 2007, le Bureau National de Gestion des Risques et des Catastrophes ignore ces protocoles validés par tous, court-circuite ses partenaires internationaux, et organise ses propres évaluations rapides. Il diffuse ensuite ses chiffres sur la catastrophe, sans validation préalable par le Comité Restreint des Intervenants dans les Catastrophes (CRIC), et met en place des programmes de réponse initiale sans relation avec les dispositions du plan d'urgence.

Comme le rappelle le père Ralibera (1966), le pays est sorti de la période de colonisation avec un fort sentiment national. Dans ce contexte, la forte ingérence de l'ancienne métropole durant la période post-coloniale génère des suspicions sur les intentions françaises, mais aussi sur celles des autres pays donateurs. Cette méfiance se retrouve dans le cadre de l'action humanitaire, tant vis-à- vis des individus que des institutions. De ce fait, le Gouvernement souhaite avoir seul le contrôle de la réponse au cyclone Fame, et ce n'est que sous la pression de ses partenaires et de l'ampleur des dégâts qu'il 
accepte finalement de lancer un appel à l'aide internationale après le passage du Cyclone Ivan.

Dans le cadre de ce second cyclone, les partenaires étrangers ne se laissent pas marginaliser et déclenchent le "plan de contingence", notamment les évaluations rapides. Des experts sont envoyés sur les zones affectées dès que les conditions le permettent afin d'évaluer la situation. Cependant le caractère parcellaire des données collectées en quelques heures, sur une zone réduite, et avec des interactions limitées avec les populations sinistrées ne leur permet pas de prétendre à une quelconque valeur scientifique. Nous sommes davantage dans le cadre d'un processus qui sert à légitimer les premières données disponibles que dans celui d'une évaluation scientifique de la crise.

Les conclusions de ces évaluations procèdent autant de l'observation de faits établis que des négociations qui entourent leurs représentations. Ainsi, pour les autorités locales, cette mission est l'occasion de faire financer par des fonds d'urgence des projets que les organisations de développement ont écartés. Du côté des Agences internationales présentes à Madagascar c'est l'occasion d'élargir leurs activités et donc d'augmenter leurs budgets. Pour des consultants en contrat court, l'adoption d'un projet peut justifier une extension de leur activité. Les données qui sont validées et les conclusions de la mission reflètent ainsi les attentes et les stratégies des différents partenaires. En conséquence, les chiffres retenus - et qui deviennent la réalité de la catastrophe - résultent autant de la pondération des différents intérêts en présence que des données collectées. C'est en fait le partage de la rente humanitaire entre le Gouvernement et les partenaires humanitaires qui se joue à ce moment-là.

Les fiches EIMA sont, quant à elles, présentées comme une panacée comme leur nom l'indique : elles peuvent être utilisées, quel que soit le type d'aléas concerné. En théorie elles sont disponibles jusqu'au niveau des fokontany ${ }^{5}$, la plus petite division administrative du territoire malgache, et, à chaque niveau, le responsable local de la gestion des risques et des catastrophes doit la remplir et la faire parvenir au Bureau National de Gestion des Risques et des Catastrophes (BNGRC) qui, ensuite, traite l'information. Cependant beaucoup d'obstacles existent qui rendent l'utilisation de ces fiches aléatoire. Différentes versions circulent, ce qui rend difficile leur consolidation dans une base de données. Elles ne sont pas présentes dans toutes les collectivités locales. Dans de nombreuses zones, il n'existe pas de responsable local, et quand ils existent, ils sont rarement formés à l'utilisation de ces fiches. De plus, peu de zones sont équipées en radios BLU; or après le passage d'un cyclone c'est le seul moyen de communication fonctionnel. Et, finalement, le BNGRC n'a pas la capacité informatique et technique de traiter les informations reçues, puisque son Secrétaire Exécutif a refusé l'offre des Nations Unies de faire sous-traiter la saisie des données collectées ainsi que leur stockage à une société privée, afin d'en garder le contrôle.

Les deux piliers de la collecte précoce de données à Madagascar, l'un issue de la Réforme Humanitaire ${ }^{6}$, le plan de contingence et ses évaluations rapides, l'autre endogène, les fiches EIMA, présentent donc de sérieux problèmes de fiabilité. En revanche, grâce aux données obtenues, la configuration humanitaire peut dès les premières 24 heures alimenter ses réseaux d'information et commencer son plaidoyer. Ces données mises en récit vont illustrer les besoins identifiés et elles vont permettre de construire la représentation humanitaire du passage d'Ivan. 


\section{La mise en discours de la crise : la bataille des mots}

représentation acceptable de la crise en cours, elles doivent être insérées dans un récit pour être partagées. En effet, la crise et ses acteurs ne deviennent publics et donc réels que lorsque leurs existences et leurs situations sont objectivées dans un discours et que ce discours est diffusé (Dechaîne 2002 : 362). Cette diffusion doit être large, répétitive et fondée sur des messages simples pour que les donateurs potentiels soient sensibilisés aux conséquences de la crise (Button 2010). Une fois que la catastrophe a été mise en discours, sa représentation acquiert une vie indépendante de sa réalité matérielle (Langumier $2008: 18$ ).

Afin de comprendre comment se mettent en place ces représentations qui finissent par devenir plus tangibles que la réalité dont elles sont le reflet, il convient de revenir sur la manière par laquelle les données sont mises en discours et utilisées parfois, de manières concurrentes. Pour ce faire, nous nous intéresserons aux différents styles utilisés pour le récit de la catastrophe, et nous mettrons en lumière comment ils correspondent à des règles et à des standards différents. Puis, nous observerons la diffusion du discours, de son émission par les médias jusqu'à sa réception par le public.

Il existe plusieurs explications possibles à la faible couverture médiatique dont a bénéficié le pays lors de la saison cyclonique précédente. Nous pouvons les regrouper en deux catégories : celles d'ordre endogène et celles d'ordre exogène. Dans la première catégorie, nous trouvons le manque d'accès des journalistes aux sources de données primaires, parfois contradictoires et leur incapacité d'en produire par manque d'accès aux zones sinistrées. Dans la seconde, nous pouvons citer le manque de demande des opinions publiques pour les événements affectant Madagascar, et en conséquence le manque de moyens financiers mis à disposition des correspondants sur place.

Afin d'assurer la fluidité et la consistance du flux d'information, les Agences des Nations unies décident de créer un relais unique entre les partenaires et les médias. Pour ce faire, l'Unicef propose au Bureau National de Gestion des Risques et des Catastrophes (BNGRC) de financer un poste de responsable des relations publiques. Cette personne a pour rôle de centraliser les informations collectées et de produire des bulletins synthétiques quotidiens présentant les chiffres cumulés de la crise (nombre de sinistrés, nombre de maisons détruites, nombre d'hectares de rizières inondées, etc.), utilisables par tous les partenaires du CRIC. Ce poste est donc situé à l'interface entre la réalité matérielle de la crise et sa narration. Dans les faits, des erreurs apparaissent dans les calculs, mais les données sont maintenues dans des marges acceptables pour les donateurs grâce au contrôle que les urgentistes exercent sur les rapports produits par le BNGRC.

Ce contrôle est notamment visible dans le cadre du cluster IEC, ou Information Éducation et Communication, qui réunit la responsable de la communication du BNGRC et les correspondants de presse sous les auspices de l'Unicef. L'Agence est ainsi en mesure de superviser son travail et (de tenter) d'influer sur les informations distribuées afin qu'elles constituent des "éléments de discours " utilisables pour quantifier et qualifier la crise. Cette tentative de mise sous tutelle est ressentie négativement par le Secrétaire du BNGRC qui lui aussi tente de l'utiliser pour promouvoir son action personnelle ainsi que celle du Gouvernement. Ces tensions et 
aspirations contradictoires se traduisent dans la manière dont le récit de la crise est construit par les différents partenaires et en fonction des différentes audiences visées.

La mise en récit d'une crise, ce qui va lui donner sa réalité, répond à des conventions établies. Les rapports de situation des organisations (sitrep) sont ainsi rédigés sur la base de canevas établis au niveau des sièges. Mais au-delà de la forme, un autre facteur intervient dans la standardisation de la couverture de la catastrophe, c'est la manière dont les faits sont mis en scène afin de sensibiliser les donateurs potentiels. C'est ce qui est appelé en communication le «storytelling» (Salmon 2007).

\section{La mise en récit : le storytelling}

Benthall (1991: 3) utilise le travail de Propp $(1928)^{7}$ pour montrer comment la communication autour des crises reprend la structure des contes de fées. Dans les articles de presse, le méchant est incarné par le cyclone Ivan, le héros est la configuration humanitaire. Le héros doit être aidé par un être surnaturel qui lui donne un pouvoir, ce sont les bailleurs de fonds qui, avec leurs dons, alimentent l'opération et permettent au héros/configuration humanitaire de résoudre le problème et de vaincre le méchant/Ivan.

30 À partir de cette trame, une approche consiste à faire débuter le récit par un événement fort par rapport auquel l'audience va devoir se positionner et relativiser sa propre expérience : «Le passage du cyclone Ivan a fait près de 190000 sans-abris. 177 personnes sont portées disparues.» (L'express.fr, 29 février 2008). Ces phrases en caractères gras qui introduisent l'article sont là pour faire réagir le lecteur. Il est pris à partie et la comparaison avec le confort dans lequel il se trouve au moment de sa lecture doit le pousser à l'empathie vis-à-vis des sinistrés. Le récit peut aussi s'articuler autour d'un témoignage individuel afin d'humaniser le récit qui est repris d'un communiqué ou d'un article à un autre.

"C'était incroyable!», se rappelle une habitante du village, Marie-Claire. « Notre maison a été emportée par le vent et nous n'avons pas pu sauver nos biens. Heureusement, il n'y a pas eu de victimes. » (UNICEF Madagascar 21 février 2008)

31 La crise n'est plus virtuelle, elle s'incarne dans Marie-Claire qui peut exprimer sa supplique (Fassin 2000). Il n'est plus question de 190000 sans-abris, statistique anonyme qui disparaît dans le flot d'informations. Le lecteur peut s'identifier à la souffrance de Marie-Claire et à la perte de sa maison; il va donc être plus réceptif à l'idée que quelque chose doit être fait.

Quand il apparaît dans la mise en récit, le bénéficiaire est décrit, contextualisé, ses représentations photographiques accompagnent les textes décrivant la crise. Il est au centre de l'histoire qui est dite, et parfois la parole lui est donnée comme pour MarieClaire et sa maison. Grâce à elle une relation se crée entre le bienfaiteur à venir et la crise. Cependant, en fonction de l'image que l'on veut véhiculer, le rôle assigné au "sinistré » varie dans le récit. Il peut être une victime impuissante que l'on doit secourir ou un agent actif de sa propre salvation et qui ne demande qu'un soutien. 


\section{La mise en image}

33 La photographie présentée en couverture de l'Appel d'Urgence représente trois enfants devant une hutte effondrée. Deux sont assis, le dernier est debout appuyé sur un morceau de bois, aucun adulte n'apparaît dans le champ de l'image. Les deux garçons ne portent qu'un short et la fillette, une blouse rose. Tous fixent l'objectif de l'appareil photographique avec un regard intense et un visage grave. Chouliaraki (2010) qualifie de "shock effect", ce type de communication qui réduit le bénéficiaire à une victime impuissante. Ici, en effet, il est fait appel au sentiment de honte et à la culpabilité de celui qui possède vis-à-vis de celui qui ne possède pas. Ce n'est pas seulement le bénéficiaire qui adresse sa demande aux bailleurs de fonds, c'est le pays tout entier. Cette photographie a été choisie par le siège de New York, pas par les équipes de terrain et encore moins par les autorités malgaches. Elle est en contradiction avec la fierté nationale. Ces enfants n'illustrent pas seulement le passage d'un cyclone et la souffrance des personnes affectées. Ils incarnent la vulnérabilité du pays tout entier et son incapacité à répondre à la crise.

Il existe une autre approche pour introduire l'image du bénéficiaire dans le discours créé autour de la crise. Elle consiste à le présenter comme un acteur à part entière. C'est la communication choisie par le Gouvernement après le passage du premier cyclone Fame. Dans les journaux pro- gouvernement aux ou politiquement neutres comme Midi Madagasikara et L'hebdo de Madagascar, les photos qui illustrent les articles consacrés à l'événement montrent les populations affectées nettoyant les villages, apportant du matériel, ou colmatant les digues avec l'aide de l'armée et du Corps de protection Civile (CPC). La figure de proue de cette version, qui présente un pays qui fait front et qui est maître de son destin, est le Président Ravalomanana lui-même. Vêtu d'un treillis militaire, il apparaît dans chaque article, là portant un sac de riz, là debout sur une digue discutant avec des villageois. Nous nous trouvons ici dans ce que Chouliaraki (2010) décrit comme un appel positiviste.

Finalement, une autre forme que peut prendre le récit et l'iconographie pour sensibiliser le lecteur est la métaphore. Les travaux de Thibodeau et Boroditsky (2011) mettent ainsi en évidence que les métaphores utilisées dans l'introduction d'un sujet conditionnent la perception que va s'en faire le lecteur ou l'auditeur. L'accroche « Ivan le terrible » de la couverture de L'Hebdo de Madagascar (22-28 février), que nous avons déjà citée, offre un bon exemple de cette démarche. Le lecteur associe le cyclone avec le Tsar Ivan le terrible, un personnage réputé pour sa violence et proche de la figure de l'ogre dans l'inconscient collectif. En conséquence, les sinistrés sont assimilés à ses victimes, et par leur caractère innocent, elles méritent d'être aidées. Cette image est encore renforcée par le sous-titre de l'article : «Malgaches dans la désolation ».

Le recours au storytelling et à la mise en image permet aux partenaires d'attirer l'attention sur la crise. Que ce soit en la personnifiant au travers d'une histoire individuelle, ou en structurant le récit pour interpeller le lecteur, la démarche est la même: optimiser la collecte de dons publics et privés afin de financer l'opération humanitaire. Plus la couverture médiatique sera dense, plus les opinions seront sensibilisées et plus les montants de contributions seront importants. Par le biais des médias (Ambrosetti 2008 : 83, Dechaîne $200: 360$ ) le passage du cyclone Ivan est passé d'une crise locale à un événement global. Cette utilisation des médias pour donner de la 
visibilité à la crise est communément appelée «l'effet CNN》 (Balabanova 2010, Robinson 2000) et son impact est synonyme de financement.

Cependant, dans le cas de Madagascar, la demande des agences de presse internationales pour les informations relatives au pays est faible. Si ce manque d'intérêt peut s'expliquer pour le monde anglo-saxon, puisque Madagascar n'a pas de lien historique avec lui, il est en revanche plus surprenant pour la France du fait du passé colonial qui lie les deux pays. En l'absence de demande et d'intérêt de la part du public, les médias lui préfèrent d'autres informations: la crise malgache est rapidement remplacée par d'autres sujets dans les médias. Au final, le déficit d'image de Madagascar à l'étranger limite l'impact de l'information mise en forme et diffusée depuis la Grande Île par les partenaires humanitaires et donc les financements.

Pour conclure, il convient de revenir sur l'importance de la maitrise des technologies de l'information, qui permettent la collecte et le traitement des données, et des techniques de communication, qui permettent de les transformer en information et de les diffuser. Hutchins (1995) postule que la capacité cognitive d'un individu est la somme des informations traitées par son corps et de celles traitées par les instruments qu'il utilise. Il prend l'exemple d'un pilote dans un cockpit et démontre qu'il convient de considérer l'individu et ses manettes de commande comme

" un » pour comprendre sa capacité cognitive à piloter l'avion. Dans ce modèle, les instruments technologiques deviennent des prothèses qui augmentent les capacités de l'être humain.

40 De même, les partenaires étrangers qui sont plus familiers avec les outils de l'information, issus comme eux $^{8}$ du paradigme occidental, les maîtrisent avec une " virtuosité » (Stoichita 2008) qui leur donne un avantage objectif sur leurs partenaires malgaches dans cette compétition pour la fabrication de la crise. C'est donc sans surprise que les médias ainsi que les bailleurs de fonds tendent à privilégier les données transmises par les organisations étrangères (Malkki 1996).

Durant la période de l'indépendance, Ralibera (1966: 57), identifiait la maitrise de la langue française, langue maternelle pour les colons, seconde langue pour les Malgaches, comme un outil de domination. De même, aujourd'hui, la meilleure maitrise des outils de l'information et de la communication est un élément de pouvoir des partenaires étrangers sur leurs homologues locaux et un moyen de contrôle des flux financiers humanitaires, à Madagascar comme sur la plupart de leurs terrains d'intervention.

Piers Robinson, “The News Media and Intervention: Triggering the Use of Air Power During Humanitarian Crises", European Journal of Communication 15, 2000, pp. 405-414.

\section{BIBLIOGRAPHIE}

David Ambrosetti, « Urgences et normalités de gestionnaires face aux violences " des autres », l'ONU et le Soudan ", Actes de la recherche en sciences sociales 174, avril, 2008, pp. 80-99. 
Ekaterina Balabanova, "Media power during humanitarian interventions: Is Eastern Europe any different from the West?”, Journal of Peace Research 47, 2010, pp. 71-82.

Jonathan Benthall, “Disasters, Donors, Magical Agencies”, Anthropology Today 7, October, 1991, p. 3.

Gregory Button, Disaster Culture: Knowledge and Uncertainty in the Wake of Human and Environmental Catastrophe, Left Coast Press, Wallnut Creek, 2010.

Lilie Chouliaraki, "Post-humanitarianism: Humanitarian communication beyond a politics of pity", International Journal of Cultural Studies 13, 2010, pp. 107.

Robert Dechaîne, "Humanitarian Space and the Social Imaginary: Médecins Sans Frontières/ Doctors Without Borders and the Rhetoric of Global Community", Journal of Communication Inquiry 26, 2002, p. 354 .

Arturo Escobar, "Anthropology and the Development Encounter: The Making and Marketing of Development Anthropology”, American Ethnologist 18, 1991, pp. 658-682.

Didier Fassin, « La supplique. Stratégies rhétoriques et constructions identitaires dans les demandes d'aide d'urgence », Annales. Histoire, Sciences Sociales 5, 2000, pp. 955-981.

Jean Fremigaci, "Echec de l'insurrection de 1947 et renouveau des antagonismes ethniques à Madagascar » in Françoise Raison Jourde et Solofo Randrianja, eds., La nation Malgache au défi de l'ethnicité, Karthala, Paris, 2002, pp. 317-341.

Clifford Geertz, "La description dense, vers une théorie interprétative de la culture", Enquête, anthropologie, histoire, sociologie 6, 1998, pp. 73-105.

Edwin Hutchins, Cognition in the wild. Cambridge, MIT Press, 1995.

Edward Keenan, Ochs Elinor, "Becoming a Competent Speaker of Malagasy" in Timothy Shopen, ed., Languages and Their Speakers, Winthrop, Cambridge (Mass.), 1979, pp. 112-158.

Julien Langumier, Survivre à l'inondation : Pour une ethnologie de la catastrophe, ENS Éditions, Lyon, 2008.

Liisa Malkki, “Speechless Emissaries: Refugees, Humanitarianism, and Dehistoricization”, Cultural Anthropology Volume 11, No. 3, August 1996, pp. 377-404.

Anthony Oliver-Smith, "Anthropological research on hazards and disasters", Annual Review of Anthropology 25, 1996, pp. 303-328.

Jean-Pierre Olivier de Sardan, Anthropologie et développement, Karthala, Paris,1995.

Françoise Raison-Jourde, Gérard Roy, Paysans, intellectuels et populisme à Madagascar : de Monja Joana à Ratsimandrava (1960-1975), Karthala, Paris, 2010.

Remy Ralibera, Vazaha et Malgaches en dialogue, Imprimerie catholique, Tananarive, 1966.

Christian Salmon, Storytelling, La machine à fabriquer des histoires et à formater les esprits, La Découverte, Paris, 2008.

Jacky Simonin, « Pour une anthropologie empirique de l'événement », Études de communication, 1999 [En ligne] 22 : 93-114, mis en ligne le 23 mai 2011, consulté le 2 janvier 2017. URL : http:// edc.revues.org/2348; DOI : 10.4000/edc.2348.

Victor Stoichita, Fabricants d'émotion, Musique et malice dans un village tsigane de Roumanie, Société d'ethnologie, Nanterre, 2008. 
Paul Thibodeau, Lera Boroditsky, "Metaphors We Think With : The Role of Metaphor in Reasoning”, PLoS ONE 6(2), 2011.

Janine Wedel, Chris Shore, Gregory Feldman et Stacy Lathrop, “Toward an Anthropology of Public Policy", The Annals of the American Academy of Political and Social Science 600, July, 2005, pp. 30-51.

Articles de presse

« Dégâts cycloniques, l'état boude la communauté internationale », La Gazette de Madagascar, 22 février2008, Tananarive.

«Cyclone à Madagascar, 23 morts », L'Express.fr, 29 février2008, http://www.lexpress.fr/ actualite/monde/cyclone-a-madagascar-83-morts_470717.html

« Aides aux sinistrés, « Ravalomanana n'a pas abandonné Tana », L'Hebdo de Madagascar, 22- 28 février2008, Tananarive.

\section{NOTES}

1. Le concept de configuration humanitaire est construit à partir de celui de configuration développementiste qu'Olivier de Sardan (1995: 5) définit comme «Un univers largement cosmopolite d'experts, de bureaucrates, de responsables d'ONG, de chercheurs, de techniciens, de chefs de projets, d'agents de terrain, qui vivent en quelque sorte du développement des autres, et mobilisent ou génèrent à cet effet des ressources matérielles et symboliques considérables ». Nous préférerons cette terminologie pour définir les acteurs impliqués dans l'aide humanitaire d'urgence à celle de « communauté humanitaire » dont les contours restent flous.

2. Organisation météorologique mondiale (http://www.wmo.int)

3. L'acronyme français BCAH n'est jamais utilisé, même dans les documents rédigés en français. C'est pourquoi nous utilisons dans ce travail l'acronyme anglais OCHA. Cette entité, créée en 1998 est un bureau du Secrétariat Général des Nations Unies, son mandat est de coordonner les opérations humanitaires qu'elles soient générées par un conflit ou un événement naturel. (Pour plus d'informa- tion voir : http://www.unocha.org)

4. www.reliefweb.int

5. Le terme fokontany recouvrait à l'origine le sens de village, il correspond aujourd'hui la division administrative de base du territoire et peut aussi décrire le quartier d'une ville.

6. Cette Réforme a été mise en place par le Comité Permanent Inter-Agences. (https:// interagencystandingcommittee.org) après l'inadéquation de la réponse humanitaire à la crise du Darfour en 2004.

7. Propp, Vladimir, 1970, Morphologieduconte. Paris, Seuil (Points / Essais).

8. La plupart des travailleurs humanitaires étrangers sont occidentaux ou ont été formés en Occident. 


\section{RÉSUMÉS}

Quand un cyclone frappe l'Asie du Sud Est, un conflit se déclenche au Moyen-Orient, ou un tremblement de terre secoue l'Amérique du Sud, ces événements ne sont pas (re)connus en tant que «crises humanitaires» avant d'avoir été qualifiés, quantifiés puis diffusés. Les individus, animaux, végétaux, lieux, objets qui participent de la « catastrophe » doivent être redéfinis dans le vocabulaire de l'humanitaire pour exister. Les individus deviennent des "sinistrés", des "sans-abris ", des « déplacés ", des « victimes »; dans un second temps, ils pourront devenir des «bénéficiaires ». Les bâtiments, les arbres, les champs, les voitures deviennent des « dégâts »; ils pourront ensuite devenir des projets. Toutes ces catégories nouvellement créées justifient la présence des programmes d'aide, de la communauté humanitaire, de ses personnels et de ses activités. Sans processus de quantification et de qualification, sans diffusion de l'information, la crise n'existe pas, et l'aide humanitaire non plus. La fabrication de la crise est donc un moment essentiel qui repose sur la collecte, la mise en forme et la diffusion de l'information. Ce processus qui repose sur la maîtrise des technologies de l'information et des techniques de la communication est aussi le théâtre de tensions et de compétitions entre les différents acteurs de l'humanitaire qui veulent le contrôler. Sur la base d'un travail ethnographique mené en 2007 et 2008 à Madagascar, ce sont ces tensions entre les humanitaires internationaux et leurs contreparties nationales lors du passage des cyclones Fame et Ivan que nous étudierons dans ce travail.

When a cyclone hits South East Asia, a conflict starts in the Middle East, or an earthquake shakes South America, these events are not known as or acknowledged to be a «humanitarian crisis» by the public until they are quantified, qualified, and shared through the media. Peoples, animals, trees, objects and places that have participated in the "disaster" must be renamed and redefined through the humanitarian vocabulary. Peoples become, "homeless", "displaced" or "victims". Soon they will become "beneficiaries". The buildings, the fields, the cars become "loss and damages". They will later on turn into "projects". All these newly created categories justify the need for international assistance and the presence of the relief community. Without the process of quantification and qualification and without the dissemination of information, the crisis does not exist and neither does the international aid. Therefore, the construction of the crisis is a crucial moment that depends on data collection, treatment, and dissemination. This process, which relies on the mastery of information technologies and information techniques, also witnesses tensions and competition between the humanitarian actors who want to control it. Based on an ethnographic work led in Madagascar between 2017 and 2018, it is these tensions, which appeared in the aftermath of cyclone Fame and Ivan between international aid workers and their national counterparts, that are analysed in this work.

Wenn ein Zyklon in Süd-Ost-Asien wütet, im Mittlereren Osten ein neuer Konflikt ausbricht, oder ein Erdbeben Südamerika erschüttert, dann sind solche Ereignisse nicht im Bewusstsein der Öffentlichkeit als «humanitäre Krise» bevor sie bestätigt, in ihrem Umfang erfasst, und durch die Medien verbreitet worden sind.

Die Menschen, Tiere, Pflanzen, Ortschaften die von der jeweiligen Katasthrophebetroffensind,werden aber erst dann wahrgenommen, wenn sie von der humanitären Seite neu definiert sind: Menschen werden zu «Hilfsbedürftigen», Geschädigten» Schutzlosen» Opfern», später dann werden sie «Empfänger von Hilfeleistugnen». Gebäude, Bäume, Felder, Fahrzeuge werden zu «Schaden» Später werden sie dann zu «Projekten» All diese Kategorisierungen erst rechtfertigen die Existenz von Hilfprogrammen, den Einsatz von Hilforganisationen mit ihren Mitarbeitern und Aktionen. 
Ohne genaue Bennung des Umfanges und der Grösse der Krise, ohne Verbereitung durch die Medien, gibt es keine Krise und damit auch keinen humanitären Hilfseinsatz. Die Darstellung solcher Krisen ist daher der entscheidende Moment, der auf der Sammlung von Daten, der Verbreitung von Information und deren Ausgestaltung basiert. Dieser Prozess, der auf der Beherrschung der Informations- und Kommunikationstechnologien beruht,istauchimmereinePlattform für Spannungen und Wettbewerb zwischen den verschiedenen Akteuren internationalen Hilforganisationen, um die Durchführung der Hilfe zu kontrollieren. Auf Basis einer ethnographischen Arbeit, ausgeführt zwischen 2007 und 2008 auf Madagaskar werden im Folgenden diese Spannungen zwischen den internationalen Hilfsorganisationen und Ihren nationalen «Mit- ( und bisweilen Gegen-)spielern nach dem Durchzug der Zyklone «Fame» und «Ivan»näher beleuchtet.

INDEX

Mots-clés : humanitaire, Madagascar, communication, technologies de l'information

Keywords : humanitarian, Madagascar, communication, information technology

Schlüsselwörter : humanitäre Hilfe, Madagaskar, Kommunikation, Informationstechnologie

\section{AUTEUR}

\section{JEAN-MARIE STRATIGOS}

Fondation Maison des Sciences de l'Homme, France 